\title{
SGLT2 inhibitors for improving hepatic fibrosis and steatosis in non-alcoholic fatty liver disease complicated with type 2 diabetes mellitus: a systematic review
}

\author{
Michael Dwinata', David Dwi Putera', Irsan Hasan², Monica Raharjo² \\ 'Faculty of Medicine, Public Health and Nursing, Gadjah Mada University, Yogyakarta, Indonesia \\ ${ }^{2}$ Hepatobiliary Division, Department of Internal Medicine, Universitas Indonesia, Cipto Mangunkusumo National General Hospital, \\ Jakarta, Indonesia
}

\begin{abstract}
Aim of the study: To evaluate the efficacy of sodium/glucose cotransporter-2 inhibitors (SGLT2i) in improving hepatic fibrosis and steatosis of non-alcoholic fatty liver disease (NAFLD) patients with type 2 diabetes mellitus (T2DM).

Material and methods: We searched CENTRAL, MEDLINE, and EMBASE and included any clinical trials involving patients with NAFLD and T2DM aged $\geqslant 18$ years comparing efficacy of SGLT2i and other antidiabetic drugs in improving fibrosis and steatosis, irrespective of publication status, year of publication, and language.

Results: Five clinical trials were included. One study reported significant improvements in the controlled attenuation parameter $314.6 \pm 61.0 \mathrm{~dB} / \mathrm{m}$ to $290.3 \pm 72.7 \mathrm{~dB} / \mathrm{m}(p=0.04)$ in the SGLT2i group measured by transient elastography. In patients with significant fibrosis, dapagliflozin treatment significantly decreased the liver stiffness measurement from $14.7 \pm 5.7 \mathrm{kPa}$ at baseline to $11.0 \pm 7.3 \mathrm{kPa}$ after 24 weeks ( $p=0.02)$. One study reported a significant decrease in liver fat content $16.2 \%$ to $11.3 \%(p<0.001)$ in the SGLT2i group compared to the control $(p<0.001)$. Three studies reported significant improvement in the liver-to-spleen ratio in the SGLT2i group after treatment $0.96(0.86-1.07)$ to 1.07 (0.98-1.14), $p<0.01,0.80 \pm 0.24$ to $1.00 \pm 0.18, p<0.001$, and $0.91(0.64-1.04)$ to $1.03(0.80-1.20), p<0.001$ respectively. All studies reported a significant decrease in alanine aminotransferase with SGLT2i.

Conclusions: SGLT2i is associated with positive effects on hepatic steatosis measured by non-invasive modalities. Further studies are needed to confirm the impact of SGLT2i on hepatic fibrosis and steatosis.
\end{abstract}

Key words: NAFLD, SGLT2 inhibitor, fibrosis, steatosis, diabetes mellitus.

Address for correspondence:

Dr. Michael Dwinata, Faculty of Medicine, Public Health and Nursing, Gadjah Mada University, Yogyakarta, Indonesia, e-mail: mdwinata@gmail.com

\section{Introduction}

Non-alcoholic fatty liver disease (NAFLD) can be defined as the presence of hepatic steatosis in the absence of other secondary causes of hepatic fat accumulation (such as heavy alcohol consumption, hypothyroidism, and drugs). It is subdivided into non-alcoholic fatty liver (NAFL) and non-alcoholic steatohepatitis (NASH). In NAFL, there is no evidence of hepatic inflammation, whereas in NASH, both hepatic steatosis and hepatic inflammation are present. NASH is histologically undistinguishable from alcoholic steatohepatitis. NAFLD is an important cause of cryptogenic cirrhosis, an important risk factor for development of hepatocellular carcinoma 
(HCC). NAFLD may also progress directly to HCC [1-3]. The worldwide prevalence of NAFLD is 6-35\% (median 20\%). It is the most common liver disorder in Western industrialized countries and may soon become the most common indication for liver transplantation $[4,5]$.

Diagnosis of NAFLD is made based on a patient's clinical history, laboratory study, imaging, and histology. Hepatic steatosis on abdominal imaging may be sufficient for diagnosis of NAFLD. However, liver biopsy is important to differentiate NASH and NAFL. NASH is confirmed when steatosis, inflammation, cellular ballooning, and fibrosis are present on liver biopsy. NASH is associated with a higher risk of disease progression compared to NAFL $[6,7]$. Non-invasive tests are also helpful to identify the presence of advanced fibrosis in NAFLD patients.

Transient elastography (TE) is an ultrasound-based method of elastography which can evaluate hepatic steatosis and liver fibrosis simultaneously by measuring the controlled attenuation parameter (CAP) and liver stiffness (LS). LS is strongly correlated with the stage of liver fibrosis assessed by liver biopsy $[8,9]$. $\mathrm{TE}$ is also more accurate than biomarkers or a scoring system in detecting significant fibrosis and cirrhosis of the liver [10]. Computed tomography (CT) is another non-invasive tool for diagnosis of NAFLD and NAFLD severity. Park et al. found that cut-off values of 0.9 and 58 Hounsfield units (HU) for liver-to-spleen attenuation ratio (L/S ratio) and liver attenuation respectively provide good sensitivity and specificity for determining the presence of $\geq 30 \%$ steatosis with CT [11].

The NAFLD fibrosis score (NFS) is a validated non-invasive tool commonly employed to identify NAFLD patients with advanced liver fibrosis. This tool utilizes six variables: age, body mass index (BMI), hyperglycemia, platelet count, and aspartate and alanine aminotransferase (AST/ALT) ratio. NFS has an area under the receiver operating curve (AUROC) of 0.85 for predicting bridging fibrosis with nodularity or cirrhosis. NFS less than -1.45 had a $90 \%$ sensitivity to exclude advanced fibrosis, while NFS more than 0.67 had a $97 \%$ specificity to identify presence of advanced fibrosis [12]. The Fibrosis-4 (FIB-4) index is another non-invasive scoring system that is used to predict advanced liver fibrosis based on age, platelet count, AST, and ALT. Imajo et al. reported that the NFS and FIB-4 index were better than other non-invasive scoring indices such as the AST to platelet ratio and AST/ALT ratio in predicting advanced fibrosis. They were as good as magnetic resonance elastography (MRE) in identifying biopsy-proven NAFLD patients with advanced fibrosis [13].

Although numerous pharmacological strategies have been evaluated or are currently in development, no phar- macotherapy has been approved for patients with NAFL and NASH $[14,15]$. Evidence for efficacy of antidiabetic medications for NAFLD is limited and conflicting. Insulin, metformin, sulphonylureas, and dipeptidyl peptidase (DPP)-4 inhibitors showed no significant effect; meanwhile, thiazolidinediones and glucagon-like peptide (GLP)-1 receptor agonists showed positive effects [16, 17].

Sodium-glucose co-transporter-2 inhibitors (SGLT2i) are a new class of oral antidiabetic drugs that promote urinary excretion of glucose, thereby reducing hyperglycemia independent of insulin secretion [18]. Diabetic patients receiving dapagliflozin, an SGLT2i, were reported to experience weight loss mainly due to fat loss with a significant reduction in the volume of abdominal visceral adipose tissue (VAT) and subcutaneous adipose tissue (SAT) [19]. Furthermore, studies in rodent models reported that SGLT2i improve histological hepatic steatosis or steatohepatitis in obese mice or rats with T2DM, suppressing development of NAFL and/or NASH [20-22].

We hypothesized that SGLT2i may cause more significant improvement of hepatic fibrosis and steatosis in NAFLD patients with T2DM compared to other antidiabetic drugs. This systematic review was performed to summarize current evidence.

\section{Material and methods}

\section{Study sample, design, and setting}

Patients with NAFLD and T2DM $\geq 18$ years old were included in this systematic review. We included any clinical trials comparing the efficacy of SGLT2 $i$ and other antidiabetic drugs for improving liver fibrosis and steatosis, irrespective of publication status, year of publication, and language.

\section{Treatment outcomes}

The primary outcome was improvement of fibrosis and/or steatosis assessed histologically or by non-invasive modalities (TE, MR spectroscopy or proton density fat fraction [PDFF], CT scan). The secondary outcomes were changes in hemoglobin $\mathrm{A}_{1 c}\left(\mathrm{HbA}_{1 \mathrm{c}}\right)$, VAT, liver enzymes (AST, ALT, and $\gamma$-glutamyl transferase [GGT]), or various markers/scores for hepatic fibrosis.

\section{Search strategy and literature review}

Two independent reviewers (MD and DDP) searched the MEDLINE, Cochrane Central Register of Controlled Trials (CENTRAL), and EMBASE journal databases from November 2019 to May 2020 (Table 1). References of the retrieved articles were perused for po- 
Table 1. Keywords for literature searching

\begin{tabular}{|c|c|c|}
\hline Journal database & Search terms & Articles \\
\hline MEDLINE & $\begin{array}{c}\text { ("Non-alcoholic fatty liver disease" or NAFLD or fatty liver) AND (SGLT2 OR "SGLT-2 } \\
\text { inhibitor" OR Dapagliflozin OR Canagliflozin OR Empagliflozin) AND (Fibrosis or } \\
\text { steatosis or histology) }\end{array}$ & 121 \\
\hline CENTRAL & $\begin{array}{c}\text { ("Non-alcoholic fatty liver disease" or NAFLD or fatty liver) AND (SGLT2 OR "SGLT-2 } \\
\text { inhibitor" OR Dapagliflozin OR Canagliflozin OR Empagliflozin) AND (Fibrosis or } \\
\text { steatosis or histology) }\end{array}$ & 30 \\
\hline EMBASE & $\begin{array}{l}\text { ("Non-alcoholic fatty liver disease" or NAFLD or fatty liver) AND (SGLT2 OR "SGLT-2 } \\
\text { inhibitor" OR Dapagliflozin OR Canagliflozin OR Empagliflozin) AND (Fibrosis or } \\
\text { steatosis or histology) }\end{array}$ & 2541 \\
\hline $\begin{array}{l}\text { A manual search of abstracts and citation index } \\
\text { from identified paper's reference list, WHOICTRP, } \\
\text { and via https://library.sydney.edu.au/ }\end{array}$ & - & 26 \\
\hline
\end{tabular}

tentially relevant studies. Abstracts and other gray literature were also included through a manual and electronic search of the clinical trial registries (e.g. WHOICTRP or World Health Organization International Clinical Trial Registry Platform) and other electronic databases.

\section{Selection of study}

Relevant studies, screened based on the title and abstract, were selected after conducting an electronic search. Studies on animals and review articles were excluded. Disagreement was resolved through discussion, failing which a third reviewer was consulted. The study selection process was plotted using a Preferred Reporting Items for Systematic Reviews and Meta-Analyses (PRISMA) flow diagram. The relevant studies were independently appraised using an Oxford Centre for Evidence-Based Medicine critical appraisal tool.

\section{Assessment of bias}

Risk of bias was independently determined using the Cochrane risk-of-bias tool. The data were then included in a table. Risk of bias was classified as low, high, or unclear. Disagreement was resolved through discussion, failing which a third reviewer was consulted.

\section{Data synthesis and analysis}

We gathered the available data from every included study in tabular fashion and we described the results narratively.

\section{Results}

One hundred twenty-one, 30, and 2541 relevant references were identified in the MEDLINE, CENTRAL, and EMBASE via Ovid databases, respectively. Twentysix of them were selected through a manual search of the references in the identified papers and other clinical trial databases. Forty-nine studies were duplicates and removed. The abstracts were also filtered, leading to the removal of 2628 studies that met the exclusion criteria for various reasons; i.e., the study did not use SGLT2i, the patients were not NAFLD patients with T2DM, the study was not a clinical trial or the study was conducted on animals. Forty-one full-text studies were assessed, and 36 studies were excluded. Five full-text studies were included in the qualitative synthesis (Fig. 1).

Four randomized controlled trials (RCT) and 1 quasiexperiment were included in the current study. Two studies added dapagliflozin $5 \mathrm{mg} /$ day to the treatment
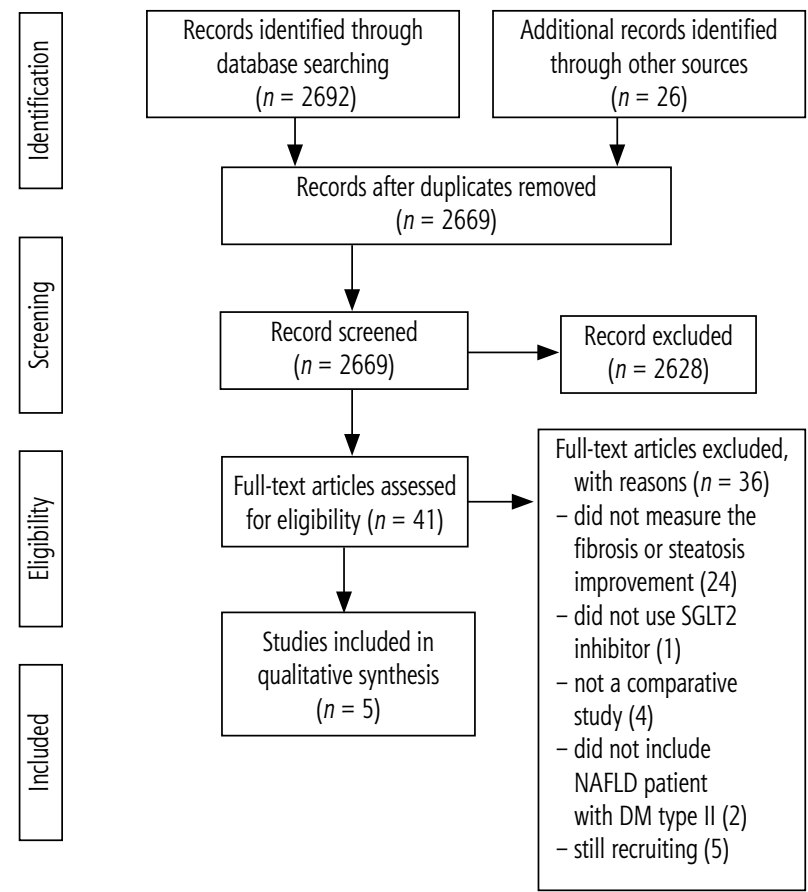

Fig. 1. PRISMA flowchart 


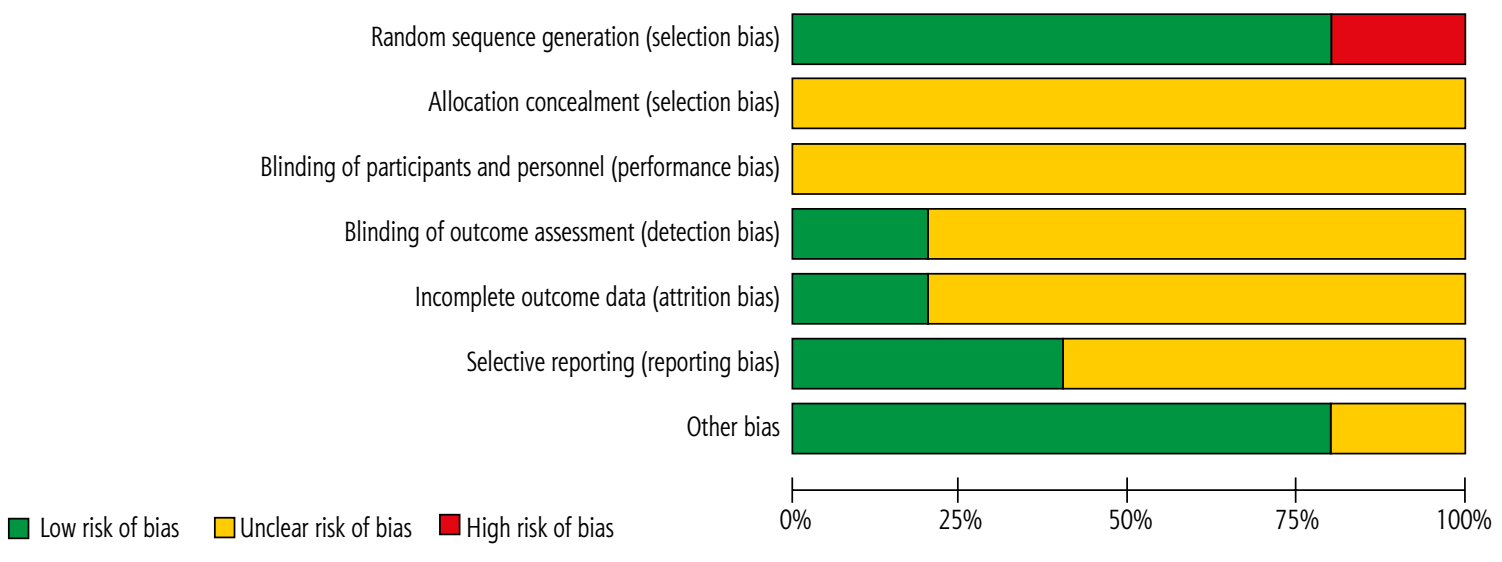

Fig. 2. Risk of bias graph of included studies

of the control group, one study added empagliflozin $10 \mathrm{mg} /$ day, one study added ipragliflozin $50 \mathrm{mg}$ /day, and one study used luseogliflozin $2.5 \mathrm{mg} /$ day [23-27]. The risk of bias is depicted in Figures 2 and 3. Overall the included studies have moderate to high risk of bias, especially in terms of allocation concealment and blinding methods. Most of the studies did not clearly mention these processes. Therefore, the quality of included studies was deemed low to moderate.

Table 2 shows the characteristics of included studies, including author, year of publication, study design, and studied population. For outcome measurement, one study used TE to measure CAP for steatosis and LS for fibrosis, one used magnetic resonance imaging de-

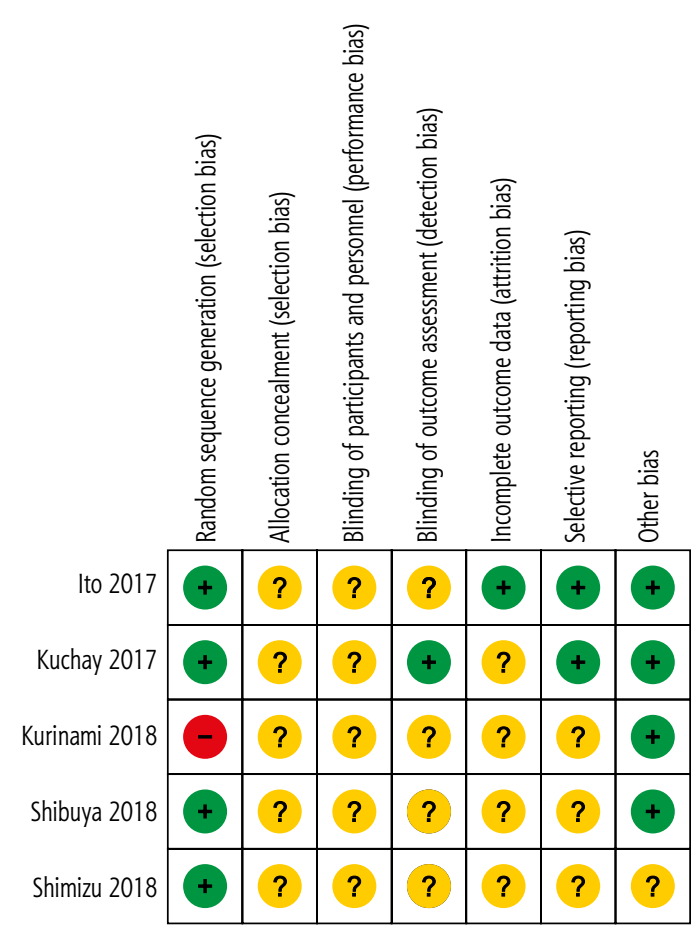

Fig. 3. Risk of bias summary of included studies rived proton density fat fraction (MRI-PDFF) to measure liver fat content, and three studies used CT scan to measure L/S ratio to quantify liver fat content. Unfortunately, no relevant comparative study performed with biopsy to assess histological improvement of NAFLD patients with T2DM after SGLT2i treatment. Table 3 is a summary table of the included studies.

All included studies showed improvement of steatosis, measured by different modalities, in NAFLD patient with T2DM after SGLT2i treatment. Only one study assessed changes in liver fibrosis after SGLT2i treatment using TE. This study showed that after additional treatment of dapagliflozin $5 \mathrm{mg}$ /day, there was a decrease in liver stiffness measurement (LSM) $(\mathrm{kPa})$ from $9.49 \pm 6.05$ to $8.01 \pm 5.78$ though not statistically significant $(p=0.05)$. In contrast, LSM in the control group increased from $7.40 \pm 3.76$ to $7.85 \pm 4.18$ $(p=0.87)$. Additionally, they divided the 33 patients who completed dapagliflozin treatment into subgroups with or without significant liver fibrosis, which were stratified according to a baseline LSM $>8.0$ or $<8.0 \mathrm{kPa}$. In the 14 patients with significant fibrosis, the LSM was decreased from $14.7 \pm 5.7 \mathrm{kPa}$ at baseline to $11.0 \pm 7.3 \mathrm{kPa}$ after 24 weeks $(p=0.02)$ [23].

Only one study assessed changes in the FIB-4 index and NFS after SGLT2i treatment. Patients in the SGLT2i group showed a decrease of the FIB-4 index from $1.32(0.74-2.10)$ to $1.27(0.77-1.91), p=0.72$; meanwhile, patients in the control group experienced an increase of the FIB- 4 index from $1.11 \pm 0.64$ to 1.17 $\pm 0.70, p=0.93$. NFS decreased in patients of both treatment groups though not statistically significantly ( $p=0.30$ and $p=0.52$ respectively) [23].

For other clinical parameters, we found that in most studies, treatment with SGLT2i was followed by a statistically significant decrease in ALT and AST compared to a statistically non-significant decrease in the control group. In most studies, GGT and $\mathrm{HbA}_{1 c}$ also decreased 
Table 2. Characteristics of included studies

\begin{tabular}{|c|c|c|c|c|c|}
\hline No. & Author & Location & Design & Publication year & Study characteristics \\
\hline \multirow[t]{3}{*}{1} & \multirow{3}{*}{$\begin{array}{c}\text { Shimizu et al. } \\
\text { [23] }\end{array}$} & \multirow[t]{3}{*}{ Japan } & \multirow[t]{3}{*}{ RCT } & \multirow[t]{3}{*}{2018} & Sample size: 57 NAFLD patients complicated with T2DM \\
\hline & & & & & Mean age: intervention group $56.2 \pm 11.5$, control group $57.1 \pm 13.8$ \\
\hline & & & & & Duration: 24 weeks \\
\hline \multirow[t]{3}{*}{2} & \multirow{3}{*}{$\begin{array}{c}\text { Kuchay et al. } \\
{[24]}\end{array}$} & \multirow[t]{3}{*}{ India } & \multirow[t]{3}{*}{ RCT } & \multirow[t]{3}{*}{2018} & Sample size: 42 NAFLD patients complicated with T2DM \\
\hline & & & & & Mean age: not mentioned \\
\hline & & & & & Duration: 20 weeks \\
\hline \multirow[t]{3}{*}{3} & \multirow{3}{*}{$\begin{array}{l}\text { Kurinami } \\
\text { et al. [25] }\end{array}$} & \multirow[t]{3}{*}{ Japan } & \multirow{3}{*}{$\begin{array}{c}\text { Quasi- } \\
\text { experiment }\end{array}$} & \multirow[t]{3}{*}{2018} & Sample size: 55 NAFLD patients complicated with T2DM \\
\hline & & & & & Mean age: $56.0 \pm 8.4$ \\
\hline & & & & & Duration: 24 weeks \\
\hline \multirow[t]{3}{*}{4} & \multirow[t]{3}{*}{ Ito et al. [26] } & \multirow[t]{3}{*}{ Japan } & \multirow[t]{3}{*}{ RCT } & \multirow[t]{3}{*}{2017} & Sample size: 66 NAFLD patients complicated with T2DM \\
\hline & & & & & Mean age: intervention group $57.3 \pm 12.1$, control group $59.1 \pm 9.8$ \\
\hline & & & & & Duration: 24 weeks \\
\hline \multirow[t]{3}{*}{5} & \multirow{3}{*}{$\begin{array}{c}\text { Shibuya et al. } \\
\text { [27] }\end{array}$} & \multirow[t]{3}{*}{ Japan } & \multirow[t]{3}{*}{ RCT } & \multirow[t]{3}{*}{2018} & Sample size: 32 NAFLD patients complicated with T2DM \\
\hline & & & & & Mean age: intervention group 51 (47-62), control group 60 (53-66) \\
\hline & & & & & Duration: 24 weeks \\
\hline
\end{tabular}

Table 3. Summary table of included studies

\begin{tabular}{|c|c|c|c|c|c|c|c|}
\hline \multirow[t]{2}{*}{ No. } & \multirow[t]{2}{*}{ Author } & \multirow[t]{2}{*}{ Intervention } & \multirow[t]{2}{*}{ Comparator } & \multicolumn{2}{|c|}{ Primary outcome } & \multicolumn{2}{|c|}{ Secondary outcomes } \\
\hline & & & & SGLT2i group & Control & SGLT2i group & Control \\
\hline 1 & $\begin{array}{c}\text { Shimizu et al. } \\
\text { [23] }\end{array}$ & $\begin{array}{c}n=33 \\
\text { Added } \\
\text { dapagliflozin } \\
5 \mathrm{mg} / \text { day }\end{array}$ & $\begin{array}{c}n=24 \\
\text { Standard } \\
\text { treatment } \\
\text { without } \\
\text { SGLT2i }\end{array}$ & $\begin{array}{c}\text { 1. Change in } \\
\text { CAP }(\mathrm{dB} / \mathrm{m}) \\
314.6 \pm 61.0 \\
\rightarrow 290.3 \\
\pm 72.7 \\
p=0.04 \\
2 . \text { Change in } \\
\text { LSM (kPa): } \\
9.49 \pm 6.05 \\
\rightarrow 8.01 \\
\pm 5.78 \\
p=0.05\end{array}$ & $\begin{array}{l}\text { 1. Change in CAP } \\
(\mathrm{dB} / \mathrm{m}) \\
306.0 \pm 34.3 \\
\rightarrow 311.3 \pm 37.3 \\
p=0.63 \\
\text { 2. Change in LSM } \\
(\mathrm{kPa}): \\
7.40 \pm 3.76 \rightarrow \\
7.85 \pm 4.18 \\
p=0.87\end{array}$ & $\begin{array}{c}\text { 1. AST (U//): } 28.0(20.5-49.8) \\
\rightarrow 27.5(17.3-31.8) \\
p<0.01 \\
\text { 2. ALT (U//): } 38.0(21.5,61.0) \\
\rightarrow 26.5(16.3,42.5) \\
p<0.001 \\
\text { 3. GGT }(\mathrm{U} / /): 47.0(28.0,88.3) \\
\rightarrow 27.0(20.5,61.5) \\
p=0.001 \\
\text { 4. HbA } 1 \text { : } 8.37 \pm 1.48 \\
\rightarrow 7.36 \pm 1.22, p<0.001 \\
\text { 5. FIB-4: } 1.32(0.74-2.10) \\
\rightarrow 1.27(0.77-1.91) \\
p=0.72 \\
\text { 6. NFS: }-0.66(-1.91 \text { to }-0.05) \\
\rightarrow-0.78(-1.66 \text { to }-0.22), \\
p=0.30\end{array}$ & $\begin{array}{c}\text { 1. AST (U/I): } 29.8 \pm 12.8 \\
\rightarrow 27.4 \pm 9.6, p=0.34 \\
\text { 2. ALT (U/I): } 33.0(24.5-46.5) \\
\rightarrow 32.0(25.0-49.3), p=0.45 \\
\text { 3. GGT (U//): } 37.5(20.0-62.3) \\
\rightarrow 32.0(22.3-50.0), \\
p=0.465 \\
\text { 4. HbA } 1 \text { : } 7.70 \pm 1.24 \\
\rightarrow 7.22 \pm 1.11, p=0.14 \\
\text { 5. FIB-4: } 1.11 \pm 0.64 \\
\rightarrow 1.17 \pm 0.70, p=0.93 \\
\text { 6. NFS: }-1.41(-2.50 \text { to }-0.46) \\
\rightarrow-1.12(-2.24 \text { to }-0.40), \\
p=0.52\end{array}$ \\
\hline 2 & $\begin{array}{c}\text { Kuchay et al. } \\
\text { [24] }\end{array}$ & $\begin{array}{c}n=22 \\
\text { Added } \\
\text { empagliglozin } \\
10 \mathrm{mg} / \text { day }\end{array}$ & $\begin{array}{l}n=20 \\
\text { Control } \\
\text { group }\end{array}$ & $\begin{array}{c}\text { Change in } \\
\text { liver fat } \\
\text { content } \\
\text { quantified } \\
\text { by MRI-PDFF, } \\
\text { average (\%) } \\
16.2(7.0) \\
\rightarrow 11.3(5.3) \text {, } \\
p<0.001\end{array}$ & $\begin{array}{c}\text { Change in liver fat } \\
\text { content quantified } \\
\text { by MRI-PDFF, } \\
\text { average (\%) } \\
16.4(7.3) \\
\rightarrow 15.5(6.7) \\
p=0.05\end{array}$ & $\begin{array}{l}\text { 1. AST (U/I): } 44.6(23.5) \\
\rightarrow 36.2(9.0), p=0.04 \\
\text { 2. ALT (U//): } 64.3(20.2) \\
\rightarrow 49.7(25.8), p<0.01 \\
\text { 3. GGT (U//): } 65.8(36.1) \\
\rightarrow 50.9(24.6), p<0.01 \\
\quad \text { 4. } \mathrm{HbA} \mathrm{A}_{1 \mathrm{l}}: 9.0(1.0) \\
\rightarrow 7.2(0.6), p<0.001\end{array}$ & $\begin{array}{c}\text { 1. AST (U/I): } 45.3(24.3) \\
\rightarrow 44.6(23.8), p=0.93 \\
\text { 2. ALT (U/I): } 65.3(40.3) \\
\rightarrow 61.6(38.4), p=0.42 \\
\text { 3. GGT (U/I): } 63.9(45.3) \\
\rightarrow 60.0(39.0), p=0.42 \\
\text { 4. } \mathrm{HbA}_{1 \mathrm{c}}: 9.1(1.4) \rightarrow 7.1(0.9), \\
\qquad<<0.001\end{array}$ \\
\hline
\end{tabular}




\begin{tabular}{|c|c|c|c|c|c|c|c|}
\hline 3 & $\begin{array}{l}\text { Kurinami } \\
\text { et al. [25] }\end{array}$ & $\begin{array}{c}n=28 \\
\text { Added } \\
\text { dapagliflozin } \\
5 \mathrm{mg} / \text { day }\end{array}$ & $\begin{array}{l}n=27 \\
\text { Control } \\
\text { group }\end{array}$ & $\begin{array}{c}\text { Change in L/S } \\
\quad \text { ratio } \\
0.96 \\
(0.86-1.07) \\
\rightarrow 1.07 \\
(0.98-1.14) \\
p<0.01\end{array}$ & $\begin{array}{c}\text { Change in } \mathrm{L} / \mathrm{S} \\
\quad \text { ratio } \\
1.08 \\
(0.90-1.10) \\
\rightarrow 1.10 \\
(0.94-1.17) \\
p=0.02\end{array}$ & $\begin{array}{c}\text { 1. AST (U/I): } 25.0 \\
(19.0-21.8) \rightarrow 20.5 \\
(19.0-26.0), p=0.01 \\
\text { 2. ALT }(\mathrm{U} / \mathrm{l}): 26.5(19.0-43.8) \\
\rightarrow 19.0(16.0-24.3), \\
p<0.01 \\
\text { 3. GGT }(\mathrm{U} / \mathrm{l}): 34.0(20.5-50.3) \\
\rightarrow 23.0(16.0-36.3), \\
p<0.01 \\
\text { 4. } \mathrm{HbA}_{1 \mathrm{lc}}: 7.6(7.3-8.2) \\
\rightarrow 6.7(6.3-7.2), p<0.01\end{array}$ & $\begin{aligned} & \text { 1. AST (U/l): } 22.0(19.5-25.0) \\
& \rightarrow 23(20.0-25.5), p=0.19 \\
& \text { 2. ALT (U/l): } 21.0(16.5-28.0) \\
& \rightarrow 20.0(15.0-26.5), p=0.85 \\
& \text { 3. GGT (U/l): } 36.0(23.0-67.5) \\
& \rightarrow 31.0(21.0-59.5), p=0.27 \\
& \quad \text { 4. } \mathrm{HbA}_{1 \mathrm{c}}: 7.7(7.3-8.2) \\
& \rightarrow 6.9(6.6-7.9), p<0.01\end{aligned}$ \\
\hline 4 & $\begin{array}{c}\text { Ito et al. } \\
\text { [26] }\end{array}$ & $\begin{array}{c}n=32 \\
\text { Added } \\
\text { ipragliflozin } \\
50 \mathrm{mg} \text { daily }\end{array}$ & $\begin{array}{c}n=34 \\
\text { Pioglitazone } \\
15-30 \mathrm{mg} \\
\text { daily }\end{array}$ & $\begin{array}{l}\text { Change in } \\
\text { L/S ratio } 0.80 \\
\pm 0.24 \\
\rightarrow 1.00 \\
\pm 0.18 \\
p<0.001\end{array}$ & $\begin{array}{c}\text { Change in } \mathrm{L} / \mathrm{S} \\
\text { ratio } 0.78 \pm 0.26 \\
\rightarrow 0.98 \pm 0.16 \\
p<0.001\end{array}$ & 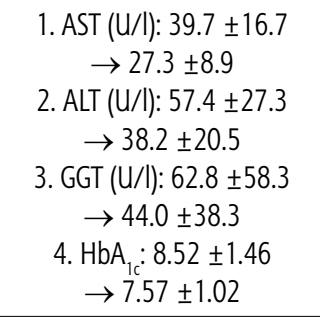 & $\begin{array}{l}\text { 1. AST (U/I): } 43.3 \pm 20.5 \\
\quad \rightarrow 32.4 \pm 15.4 \\
\text { 2. ALT (U/I): } 53.1 \pm 26.6 \\
\quad \rightarrow 36.8 \pm 15.1 \\
\text { 3. GGT (U/I): } 71.6 \pm 54.1 \\
\quad \rightarrow 48.8 \pm 61.2 \\
\text { 4. } \mathrm{HbA}_{1 \mathrm{c}}: 8.28 \pm 1.38 \\
\quad \rightarrow 7.07 \pm 0.89\end{array}$ \\
\hline 5 & $\begin{array}{l}\text { Shibuya } \\
\text { et al. [27] }\end{array}$ & $\begin{array}{l}\quad n=16 \\
\text { Luseogliflozin } \\
2.5 \mathrm{mg} \text { daily }\end{array}$ & $\begin{array}{c}n=16 \\
\text { Metformin } \\
1500 \mathrm{mg} \\
\text { daily }\end{array}$ & $\begin{array}{c}\text { Change in } \mathrm{L} / \mathrm{S} \\
\quad \text { ratio } \\
0.91(0.64 \\
1.04) \rightarrow 1.03 \\
(0.80,1.20) \\
p<0.001\end{array}$ & $\begin{array}{c}\text { Change in L/S } \\
\text { ratio } \\
0.99(0.81,1.12) \\
\rightarrow 0.85 \\
(0.68,1.00) \\
p=0.02\end{array}$ & $\begin{array}{l}\text { 1. } \operatorname{ALT}(\mathrm{U} / \mathrm{l}): 49.5(31-70) \\
\rightarrow 31(26-55), p=0.06 \\
\text { 2. } \mathrm{HbA}_{\mathrm{lc}}: 7.8(7.2-7.9) \\
\rightarrow 6.5(6.4-7.0), p<0.01\end{array}$ & $\begin{array}{c}\text { 1. ALT (U/I): } 39(23-56) \\
\rightarrow 39(27-51), p=0.52 \\
\text { 2. } \mathrm{HbA}_{1 c}: 7.4(6.9-7.7) \\
\rightarrow 7.3(6.7-7.6), p=0.36\end{array}$ \\
\hline
\end{tabular}

significantly after SGLT2i treatment compared to a nonsignificant decrease in the control group.

Unfortunately, most of the studies only performed statistical analysis comparing baseline values and values after a follow-up period of 20 or 24 weeks and did not perform statistical analysis to measure the statistical difference between the SGLT2i group and the control group. Only one study, by Ito et al., compared the difference of outcomes between ipragliflozin and control. The difference however was not statistically significant [26].

\section{Discussion}

Regardless of the different SGLT2i and control group, all five studies showed that SGLT2i treatment was associated with improvements in hepatic steatosis of NAFLD patients complicated with T2DM. The mechanisms involved in SGLT2i associated improvement of hepatic fibrosis and steatosis remain unclear. An animal study showed that empagliflozin treatment improved hepatic steatosis, inflammation, and fibrosis assessed histologically in a mouse model with NASH and diabetes, thereby preventing progression of liver disease [20]. Human studies produced similar results. In a prospective, preliminary study by Akuta et al., after 24 weeks of treatment with SGLT2i, all 5 NAFLD patients with T2DM showed improvement of histopatho- logical features in serial liver biopsy [28]. More recently, Akuta et al. also reported improvement of histopathological features in all 9 NAFLD patients with T2DM after SGLT2i treatment. Furthermore, improved BMI, waist circumference, glucose metabolism, liver serological markers, and TE findings were also observed in these patients [29]. It is not yet known whether the beneficial effects of SGLT2i in NAFLD are mediated through improvements of associated metabolic abnormalities or a direct effect of SGLT2i.

Pioglitazone, an oral antidiabetic drug belonging to the thiazolidinedione class, also demonstrated beneficial effects in NAFLD patients. Ito et al. found that ipragliflozin and pioglitazone exerted beneficial effects on NAFLD that were identical in the 24-week trial period. Amelioration of hepatic steatosis evaluated using L/S ratio, reduced ALT and AST, lowering of other NAFLD parameters, and improved glycemic parameters were observed. However, compared with pioglitazone alone, patients receiving ipragliflozin experienced significant reduction in body weight and abdominal fat [26].

To the best of our knowledge, this is the first systematic review which assesses the efficacy of SGLT2i for improving hepatic fibrosis and steatosis in NAFLD patients complicated with T2DM. A previous review by Scheen also included studies comparing SGLT2i with placebo or other antidiabetic drugs but did not aim to study the improvement of hepatic fibrosis and steatosis in study 
outcomes [30]. The review also did not include 2 recent studies by Kurinami et al. and Shimizu et al. [23, 25].

One of the limitations of this study is that we did not include studies which use placebo as a comparison. Thus, the control group encompasses multiple variations of treatment and dose intensification. We performed a preliminary literature search but only found one RCT comparing SGLT2i and placebo with a relatively short duration of follow-up [31]. Secondly, we found no comparative study which performed liver biopsy to assess changes in hepatic fibrosis and steatosis. Thirdly, due to differences in the type and dose of SGLT2i, we did not perform quantitative analysis. Lastly, all the included clinical trials of this study have a relatively small sample size.

\section{Conclusions}

We found that SGLT2i treatment is associated with positive effects on hepatic steatosis measured by non-invasive modalities. Histological assessment by performing biopsy would be the best method to confirm this result. SGLT2i also significantly improves alanine aminotransferase in NAFLD patients with T2DM. With this result, we recommend that basic and clinical research be carried out to reveal the mechanisms of action underlying this positive effects. We also strongly endorse more RCTs comparing SGLT2i treatment and placebo in NAFLD patients with T2DM.

\section{Disclosure}

The authors declare no conflict of interest.

\section{References}

1. Caldwell SH, Crespo DM. The spectrum expanded: cryptogenic cirrhosis and the natural history of non-alcoholic fatty liver disease. J Hepatol 2004; 40: 578-584.

2. Piscaglia F, Svegliati-Baroni G, Barchetti A, et al. Clinical patterns of hepatocellular carcinoma in nonalcoholic fatty liver disease: a multicenter prospective study. Hepatology 2016; 63: 827-838.

3. Ludwig J, Viggiano TR, McGill DB, et al. Nonalcoholic steatohepatitis: Mayo Clinic experiences with a hitherto unnamed disease. Mayo Clin Proc 1980; 55: 434-438.

4. Younossi ZM, Stepanova M, Afendy M, et al. Changes in the prevalence of the most common causes of chronic liver diseases in the United States from 1988 to 2008. Clin Gastroenterol Hepatol 2011; 9: 524-530.

5. Charlton MR, Burns JM, Pedersen RA, et al. Frequency and outcomes of liver transplantation for nonalcoholic steatohepatitis in the United States. Gastroenterology 2011; 141: 1249-1253.

6. Kleiner DE, Brunt EM, Van Natta M, et al. Design and validation of a histological scoring system for nonalcoholic fatty liver disease. Hepatology 2005; 41: 1313-1321.
7. Juluri R, Vuppalanchi R, Olson J, et al. Generalizability of the nonalcoholic steatohepatitis Clinical Research Network histologic scoring system for nonalcoholic fatty liver disease. J Clin Gastroenterol 2011; 45: 55-58.

8. Sandrin L, Fourquet B, Hasquenoph JM, et al. Transient elastography: a new noninvasive method for assessment of hepatic fibrosis. Ultrasound Med Biol 2003; 29: 1705-1713.

9. Castera L. Diagnosis of non-alcoholic fatty liver disease/nonalcoholic steatohepatitis: non-invasive tests are enough. Liver Int 2018; 38 Suppl 1: 67-70.

10. Piekarski J, Goldberg HI, Royal SA, et al. Difference between liver and spleen CT numbers in the normal adult: its usefulness in predicting the presence of diffuse liver disease. Radiology 1980; 137: 727-729.

11. Park SH, Kim PN, Kim KW, et al. Macrovesicular hepatic steatosis in living liver donors: use of CT for quantitative and qualitative assessment. Radiology 2006; 239: 105-112.

12. Kaswala DH, Lai M, Afdhal NH. Fibrosis assessment in nonalcoholic fatty liver disease (NAFLD) in 2016. Dig Dis Sci 2016; 61: 1356-1364.

13. Imajo K, Kessoku T, Honda Y, et al. Magnetic resonance imaging more accurately classifies steatosis and fibrosis in patients with nonalcoholic fatty liver disease than transient elastography. Gastroenterology 2016; 150: 626-637.

14. Stefan N, Haring HU, Cusi K. Non-alcoholic fatty liver disease: causes, diagnosis, cardiometabolic consequences, and treatment strategies. Lancet Diabetes Endocrinol 2018; 7: 313-324.

15. Sumida Y, Yoneda M. Current and future pharmacological therapies for NAFLD/NASH. J Gastroenterol 2018; 53: 362-376.

16. Iogna Prat L, Tsochatzis EA. The effect of antidiabetic medications on non-alcoholic fatty liver disease (NAFLD). Hormones (Athens) 2018; 17: 219-229.

17. Tacelli M, Celsa C, Magro B, et al. Antidiabetic drugs in NAFLD: the accomplishment of two goals at once? Pharmaceuticals (Basel) 2018; 11: 121-140.

18. Tahrani AA, Barnett AH, Bailey CJ. SGLT inhibitors in management of diabetes. Lancet Diabetes Endocrinol 2013; 1: 140-151.

19. Bolinder J, Ljunggren Ö, Kullberg J, et al. Effects of dapagliflozin on body weight, total fat mass, and regional adipose tissue distribution in patients with type 2 diabetes mellitus with inadequate glycemic control on metformin. J Clin Endocrinol Metab 2012; 97: 1020-1031.

20. Jojima T, Tomotsune T, Iijima T, et al. Empagliflozin (an SGLT2 inhibitor), alone or in combination with linagliptin (a DPP-4 inhibitor), prevents steatohepatitis in a novel mouse model of non-alcoholic steatohepatitis and diabetes. Diabetol Metab Syndr 2016; 8: 45-56.

21. Tahara A, Kurosaki E, Yokono M, et al. Effects of SGLT2 selective inhibitor ipragliflozin on hyperglycemia, hyperlipidemia, hepatic steatosis, oxidative stress, inflammation, and obesity in type 2 diabetic mice. Eur J Pharmacol 2013; 715: 246-255.

22. Komiya C, Tsuchiya K, Shiba K, et al. Ipragliflozin improves hepatic steatosis in obese mice and liver dysfunction in type 2 diabetic patients irrespective of body weight reduction. PLoS One 2016; 11: e0151511.

23. Shimizu M, Suzuki K, Kato K, et al. Evaluation of the effects of dapagliflozin, a sodium-glucose co-transporter-2 inhibitor, on hepatic steatosis and fibrosis using transient elastography in patients with type 2 diabetes and non-alcoholic fatty liver disease. Diabetes Obes Metab 2018; 21: 285-292.

24. Kuchay MS, Krishan S, Mishra SK, et al. Effect of empagliflozin on liver fat in patients with type 2 diabetes and non-alcoholic 
fatty liver disease: a randomized controlled trial (E-LIFT trial). Diabetes Care 2018; 41: 1801-1808.

25. Kurinami N, Sugiyama S, Yoshida A, et al. Dapagliflozin significantly reduced liver fat accumulation associated with a decrease in abdominal subcutaneous fat in patients with inadequately controlled type 2 diabetes mellitus. Diabetes Res Clin Pract 2018; 142: 254-263.

26. Ito D, Shimizu S, Inoue $\mathrm{K}$, et al. Comparison of ipragliflozin and pioglitazone effects on nonalcoholic fatty liver disease in patients with type 2 diabetes: a randomized, 24-week, open-label, active-controlled trial. Diabetes Care 2017; 40: 1364-1372.

27. Shibuya T, Fushimi N, Kawai M, et al. Luseogliflozin improves liver fat deposition compared to metformin in type 2 diabetes patients with non-alcoholic fatty liver disease: a prospective randomized controlled pilot study. Diabetes Obes Metab 2018; 20: 438-442.

28. Akuta N, Watanabe C, Kawamura Y, et al. Effects of a sodiumglucose cotransporter 2 inhibitor in nonalcoholic fatty liver disease complicated by diabetes mellitus: preliminary prospective study based on serial liver biopsies. Hepatol Commun 2017; 1: 46-52.

29. Akuta N, Kawamura Y, Watanabe C, et al. Impact of sodium glucose cotransporter 2 inhibitor on histological features and glucose metabolism of non-alcoholic fatty liver disease complicated by diabetes mellitus. Hepatol Res 2019; 49: 531-539.

30. Scheen AJ. Beneficial effects of SGLT2 inhibitors on fatty liver in type 2 diabetes: a common comorbidity associated with severe complications. Diabetes Metab 2019; 45: 213-223.

31. Eriksson JW, Lundkvist P, Jansson PA, et al. Effects of dapagliflozin and n-3 carboxylic acids on non-alcoholic fatty liver disease in people with type 2 diabetes: a double-blind randomised placebo controlled study. Diabetologia 2018; 61: 1923-1934. 